\title{
A Cardiac Sodium Channel Mutation Associated with Epinephrine-Induced Marked QT-Prolongation
}

\author{
Mohamad El Moheb ${ }^{1}$ and Marwan Refaat ${ }^{2}$ \\ ${ }^{1}$ Massachusetts General Hospital \\ ${ }^{2}$ American University of Beirut Medical Center
}

May 18, 2020

\section{A Cardiac Sodium Channel Mutation Associated with Epinephrine-Induced Marked QT- Prolongation}

Mohamad N. El Moheb MD ${ }^{1}$, Marwan M. Refaat MD ${ }^{2}$

${ }^{1}$ Division of Trauma Emergency Surgery and Surgical Critical Care, Massachusetts General Hospital, Boston, Massachusetts - USA

${ }^{2}$ Division of Cardiology, Department of Internal Medicine, American University of Beirut Medical Center Beirut, Lebanon

Running Title: SCN5A mutation associated with epinephrine-induced LQTS

Words (excluding references): 746

Disclosures: None

Funding: None

Keywords: Long QT Syndrome, Genetics, Variants, Cardiac Arrhythmias, Cardiovascular Diseases Correspondence:

Marwan M. Refaat, MD, FACC, FAHA, FHRS, FASE, FESC, FACP, FRCP

Associate Professor of Medicine

Director, Cardiovascular Fellowship Program

Department of Internal Medicine, Cardiovascular Medicine/Cardiac Electrophysiology

Department of Biochemistry and Molecular Genetics

American University of Beirut Faculty of Medicine and Medical Center

PO Box 11-0236, Riad El-Solh 1107 2020- Beirut, Lebanon

Fax: +961-1-370814

Clinic: +961-1-350000/+961-1-374374 Extension 5800

Office: +961-1-350000/+961-1-374374 Extension 5353 or Extension 5366 (Direct)

Email: mr48@aub.edu.lb 
The hereditary long QT syndrome (LQTS) is an important cause of polymorphous ventricular tachycardia (torsades de pointes) and sudden cardiac death in otherwise young and healthy individuals. Clinically, this condition is caused by delayed ventricular repolarization and manifests as an abnormally prolonged QT interval on the electrocardiogram (ECG). The most common subtypes of LQTS are LQT1, LQT2, and LQT3 (1-10). The life-threatening arrhythmias occur most frequently during exercise in LQT1, upon auditory stimulation or emotional stress in LQT2, and at rest or during sleep in LQT3 (11). Patients with LQT1 have a mutation in the KCNQ1 gene which codes for the subunit of the slow outward potassium current channel $\left(\mathrm{I}_{\mathrm{Ks}}\right)$ while patients with LQT3 have a mutation in the SCN5A gene, which codes for the cardiac voltage-dependent sodium channel $\left(\mathrm{I}_{\mathrm{Na}}\right)$ (12). LQT1-affected individuals are more vulnerable to $\beta$-adrenergic modulation than LQT3-affected individuals. Exercise and epinephrine-infusion ECG tests are therefore useful in differentiating between the LQTS subtypes and optimizing therapeutic strategies in order to prevent sudden cardiac death. While beta-blockers have been established as the standard of care for the treatment of the LQT1 and LQT2 subtypes, their use in LQT3 remains controversial $(13,14)$. A new missense mutation has been recently identified in the SCN5A-encoding $\mathrm{I}_{\mathrm{NA}}$ channels and was found to be associated with sinus node dysfunction and epinephrine-induced QT prolongation (1). This atypical phenotype of LQT3 has so far been observed in only one patient. Whether other mutations exist that can cause a similar manifestation has yet to determined.

In the current issue of the Journal of Cardiovascular Electrophysiology, Nakajima et al. describe a family with LQT3 that exhibited epinephrine-induced marked QT prolongation. The SCN5A V1667I mutation was found to be responsible for this atypical phenotype which resulted in prolongation of the QT interval in the proband as well as in family members carrying the mutation. The SCN5A V1667I mutation is a gain of function mutation located in domain IV-segment 5 (DIV-S5) of the sodium channel encoding SCN5A gene. To elucidate the pathophysiology of the disease, the authors transfected a human kidney cell line (tsA201) to induce expression of wild-type and mutated sodium channels and measured the membrane sodium currents $\left(\mathrm{I}_{\mathrm{NA}}\right)$. They showed that SCN5A V1667I mutation was associated with larger $\mathrm{I}_{\mathrm{NA}}$ peak density, depolarizing shift in steady-state inactivation (SSI) leading to increased window current, and accelerated recovery from depolarization. Additionally, an increased hump in the $\mathrm{I}_{\mathrm{NA}}$ of V1667I mutant cells (V1667I$\mathrm{I}_{\mathrm{NA}}$ ) was observed during a ramp pulse protocol consistent with increased window current. There was no difference in fast inactivation rate and steady-state activation between the V1667I-I $\mathrm{IA}_{\mathrm{NA}}$ and wild-type $\mathrm{I}_{\mathrm{NA}}\left(\mathrm{WT}-\mathrm{I}_{\mathrm{NA}}\right)$. The authors further examined the effects of protein kinase A (PKA) activation on V1667I$\mathrm{I}_{\mathrm{NA}}$ to mimic the effect of epinephrine. PKA activation resulted in a less significant hyperpolarizing shift in SSI in V1667I-I $\mathrm{I}_{\mathrm{NA}}$ compared to WT-I $\mathrm{I}_{\mathrm{NA}}$ leading to increased window current. Additionally, V1667I mutation was found to be associated with accelerated recovery from depolarization, and increased hump during ramp pulse protocol in the setting of PKA activation. Chen et al. have also reported the case of an individual with a mutation in SCN5A who exhibited marked QT-prolongation after epinephrine infusion (1). However, contrary to the SCN5A V1667I mutation described by Nakajima et al, the SCN5A V2016M defect was a loss of function mutation causing a decrease in $\mathrm{I}_{\mathrm{NA}}$ peak density. The clinical manifestations of the SCN5A mutations described by Chen et al. and Nakajima et al. are more comparable to individuals with the LQT1 subtype than those with the LQT3 subtype. Therefore, it should be considered whether certain patients with SCN5A would benefit from beta-blocker therapy.

Overall, the authors should be commended on their efforts to describe for the first time a family with the SCN5A V1667I mutation and show that this mutation is associated with epinephrine-induced marked QT prolongation. The authors have also provided important insight into the electrophysiological properties of the mutant channels and the structure-function relationship of SCN5A. Further studies are needed to elucidate the precise molecular mechanisms of PKA activation on WT-INa and V1667I-INa. The results of this study have important clinical implications. The efficacy of beta-blockers for the treatment of LQTS has so far only been proven for the LQT1 and LQT2 subtypes, with conflicting results for the LQT3 subtype $(13,14)$. Given the marked QT prolongation in response to epinephrine infusion in carriers of the SCN5A V1667I mutation, certain LQT3 patients may benefit from beta-blocker therapy. Future studies should clarify whether beta-blockers are effective in these patients. 


\section{REFERENCES}

1. Chen J, Makiyama T, Wuriyanghai Y, Ohno S, Sasaki K, Hayano M, et al. Cardiac sodium channel mutation associated with epinephrine-induced QT prolongation and sinus node dysfunction. Heart Rhythm. 2016;13(1):289-98.

2. Itoh T, Tanaka T, Nagai R, Kamiya T, Sawayama T, Nakayama T, et al. Genomic organization and mutational analysis of HERG, a gene responsible for familial long QT syndrome. Hum Genet. 1998;102(4):435-9.

3. Wang Q, Curran ME, Splawski I, Burn TC, Millholland JM, VanRaay TJ, et al. Positional cloning of a novel potassium channel gene: KVLQT1 mutations cause cardiac arrhythmias. Nat Genet. 1996;12(1):17-23.

4. Priori SG, Wilde AA, Horie M, Cho Y, Behr ER, Berul C, et al. Executive summary: HRS/EHRA/APHRS expert consensus statement on the diagnosis and management of patients with inherited primary arrhythmia syndromes. Europace. 2013 Oct;15(10):1389-406.

5. Refaat MM, Hotait M, Tseng ZH: Utility of the Exercise Electrocardiogram Testing in Sudden Cardiac Death Risk Stratification. Ann Noninvasive Electrocardiol 2014; 19(4): 311-318.

6. Refaat MM, Hotait M, London B: Genetics of Sudden Cardiac Death. Curr Cardiol Rep Jul 2015; 17(7): 606 .

7. Steffensen AB, Refaat MM, David JP, Mujezinovic A, Calloe K, Wojciak J, et al. High Incidence of Functional Ion Channel Abnormalities in a Consecutive Long QT Syndrome Cohort with Genetic Variants of Unknown Significance. Sci Rep Jun 2015; 5: 10009.

8. Hammami Bomholtz S, Refaat M, Buur Steffensen A, David J, Espinosa K, Nussbaum R, et al. Functional phenotype variations of two novel KV7.1 mutations identified in patients with Long QT syndrome. Pacing Clin Electrophysiol Feb 2020; 43 (2): 210-216.

9. Refaat MM, El Hage L, Buur Steffensen A, Hotait M, Schmitt N, Scheinman MM, et al. Iron Overload Leading to Torsades de Pointes in $\beta$-Thalassemia and Long QT syndrome. Card Electrophysiol Clin Mar 2016; 8(1): 247-56.

10. Poulsen KL, Hotait M, Calloe K, Klaerke DA, Rebeiz A, Nemer G, et al. The mutation p.T613A in the pore helix of the KV11.1 potassium channel is associated with long QT syndrome. Pacing Clin Electrophysiol Nov 2015; 38(11):1304-9.

11. Schwartz PJ, Priori SG, Spazzolini C, Moss AJ, Vincent GM, Napolitano C, et al. Genotype-phenotype correlation in the long-QT syndrome: gene-specific triggers for life-threatening arrhythmias. Circulation. 2001;103(1):89-95.

12. Kurokawa J, Chen L, Kass RS. Requirement of subunit expression for cAMP-mediated regulation of a heart potassium channel. Proceedings of the National Academy of Sciences. 2003;100(4):2122-7.

13. Priori SG, Napolitano C, Schwartz PJ, Grillo M, Bloise R, Ronchetti E, et al. Association of long QT syndrome loci and cardiac events among patients treated with beta-blockers. Jama. 2004;292(11):1341-4.

14. Shimizu W, Moss AJ, Wilde AA, Towbin JA, Ackerman MJ, January CT, et al. Genotype-phenotype aspects of type 2 long QT syndrome. J Am Coll Cardiol. 2009;54(22):2052-62. 